
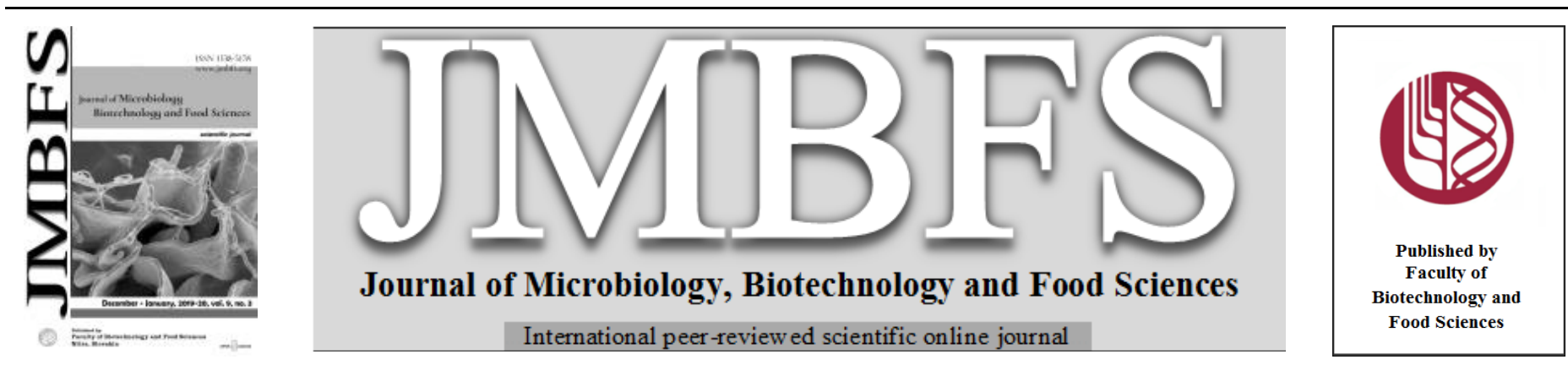

\title{
IDENTIFICATION OF MICROBIAL COMMUNITY COLONIZING THE GUT OF DYSDERCUS CINGULATUS FABRICIUS (HEMIPTERA: PYRRHOCORIDAE)
}

\author{
Shama Uzmi ${ }^{1}$, C. Shruthi Sureshan ${ }^{1,3}$, Sewali Ghosh ${ }^{2}$ \& S. K. M. Habeeb ${ }^{1,3^{*}}$
}

\section{Address(es):}

${ }^{1}$ Department of Bioinformatics, School of Bioengineering, SRM Institute of Science and Technology, Kattankulathur Campus, Chennai - 603203.

${ }^{2}$ Department of Advance Zoology and Biotechnology, Guru Nanak College, Velachery, Chennai - 600042.

${ }^{3}$ Entomoinformatics Lab, Department of Genetic Engineering, School of Bioengineering, SRM Institute of Science and Technology, Kattankulathur Campus, Chennai 603203.

*Corresponding author: habeeb_skm@yahoo.co.in , habeeb.m@ktr.srmuniv.ac.in

doi: $10.15414 /$ jmbfs.2019/20.9.3.496-501

\section{ARTICLE INFO}

Received 18. 10. 2018

Revised 10. 5. 2019

Accepted 12. 5. 2019

Published 1. 12. 2019

Regular article

OPEN $\partial_{\text {ACCESS }}$

\begin{abstract}
Red cotton stainer Dysdercus cingulatus sucks the sap of cotton plant and hence considered as one of the serious pests of cotton across the globe. Gut microbial community of this pest was studied using 16srRNA variable regions (V3 \& V4) using Illumina MiSeq technology. Totally 11, 0,797 reads were obtained which were processed using QIIME pipeline. This study resulted in the identification of gut microbiota of $D$. cingulatus categorized into 34 different phyla, 88 classes, 132 classes, 206 families and 336 genera. Phylum level taxonomic classification identifies bacteria predominantly from Proteobacteria (46.7\%), Actinobacteria (25.7\%) and Firmicutes (18\%). Species from the genus Coriobacterium, Bifidobacterium, Corynebacterium, Klebsiella \& Pseudomonas are most abundant in the gut of $D$. cingulatus. Insights into the gut can help us to understand the role of microorganism which dismantles the plant.
\end{abstract}

Keywords: Cotton Pest, Dysdercus cingulatus, 16s rRNA, Gut Microbiome, Bioinformatics, NGS MiSeq

\section{INTRODUCTION}

Dysdercus cingulatus (Hemiptera: Lygaeidae) is one among 340 species of Pyrrhocoridae family. It is a sucking pest and a common cotton stainer feeding on flower buds sand seeds; which leads to early shedding of lower buds and reduces the quality of seeds (Singh., 1924). D. cingulatus is highly mobile and while searching food it easily gets adapted to different hosts; thereby it is more challenging to control the pest when it is abundant and mobile (Maxwell-Lefroy, 1906). Inhabitations in different ecological niches flourish insects with a variety of microbes participating in Plant-Microbe-Insect (PMI) interaction. Transmission of pathogens to plants via insects (disease transmitting vector) is an example of PMI interaction (Bennett, 2013). Similarly, D. cingulatus acts as a vector or transmitting fungal pathogens to cotton plants. The insect carries fungal spores externally on the mouth parts and transmits through proboscis on the external surfaces of the cotton bolls. D. cingulatus transmits Nematospora gossypii that causes internal boll disease in cotton plants (Ahmad \& Schaefer, 1987). Another member of this genus Nematospora coryli associated with dry cultivated citrus fruit has been isolated and identified from Australia (Shivas et al., 2005).

Identification of species across different life forms using molecular approaches has been a handy tool. This is facilitated by various universal barcodes such as 16s rRNA (Bacteria) (Janda \& Abbott, 2007) and Cytochrome Oxidase subunit I, COI (Eukaryotes) (Kress \& Erickson, 2008). The 16S rRNA sequence has been a universal code in bacteria and an efficient method to identify microbial species from different environments without direct culturing of the bacterial sample. There are nine hyper variable regions in $16 \mathrm{~S}$ rRNA sequence (Srinivasan et al., 2015) that are subsequently used for bacterial identification (Huse et al., 2008).

Characterization of the gut microbial community of Pyrrhocoris apterus and Dysdercus fasciatus revealed the presence of majorly unusual nutrition mutualist Actinobacteria of genera Coriobacterium and Gordonibacter (Hassan Salem, Kreutzer, Sudakaran, \& Kaltenpoth, 2012). Firmicutes such as Clostridium and several other Gamma Proteobacteria also harbor the gut. In Pyrrhocoridae, Actinobacteria provides nutritional support by supplementing vitamins that are unavailable to the insect through malvale food source. Similar studies conducted on Halyomorpha halys (Hemiptera: Pentatomidae) to find the relationship of gut bacteria to the host health, development and fecundity also indicate negative impact on the insect if deprived of gut bacteria (Taylor, Coffey, DeLay, \&
Dively, 2014). The purpose of this study was to identify gut bacteria of Dysdercus cingulatus, a cotton stainer using 16S rRNA V3-V4 regions by Illumina MiSeq NGS technology. An insight into the microbial world residing in the gut of $D$. cingulatus can be explored for pest control management.

\section{MATERIALS AND METHODS}

\section{Insect sampling and gut extraction}

Insects collected in the month of September, 2015 from cotton field of Tindivanam, Tamil Nadu, India, were reared on cotton bolls under laboratory conditions. Identification and characterization of the sample to be $D$. cingulatus based on morphological features was done by an expert entomologist from The Department of Zoology, Guru Nanak College, Chennai - India. Prior to dissection, outer surface of insect was sterilized with $70 \%$ ethanol and rinsed twice in 1X Phosphate Buffer Saline (PBS). Midguts dissected from five $D$. cingulatus was homogenized in $180 \mu \mathrm{PBS}$ buffer using a micropestle under a sterile environment (Xiang et al., 2012).

\section{S rRNA gene sequencing, clustering and sequencing}

The genomic DNA was extracted using DNeasy Blood \& Tissue Kit (Qiagen) following manufacturer's protocol with few modifications. Insect homogenateproteinase $\mathrm{K}$ buffer mix was incubated at $37^{\circ} \mathrm{C}$ for $12 \mathrm{hrs}$ for better yield. To check the integrity and purity, extracted DNA was loaded on to $0.8 \%$ agarose gel and $1 \mu \mathrm{l}$ of the sample was loaded on NanoDrop 8000 spectrophotometer for determining A260/280 ratio respectively. The DNA was quantified using Qubit $\mathbb{}$ 2.0 Fluorometer.

Amplicon libraries were prepared using Nextera XT index Kit (Illumina Inc.) as per the 16S Metagenomics Sequencing Library preparation protocol Universal primers were used for the amplifying V3-V4 region of 16S rDNA gene (Table 1) using following reaction conditions: initial denaturation at $94^{\circ} \mathrm{C}$ for 3 minutes, followed by 30 cycles of denaturation at $94^{\circ} \mathrm{C}$ for 1 minute, annealing at $52^{\circ} \mathrm{C}$ for 1 minute and elongation at $72^{\circ}$ for 1 minute and followed by final elongation at $72^{\circ}$ for $10 \mathrm{mins}$ for identification of bacteria and (Takahashi, Tomita, Nishioka, Hisada, \& Nishijima, 2014). 
Table 1 List of Primers used for the amplification of V3-V4 hyper variable region

\begin{tabular}{llcc}
\hline \multicolumn{1}{c}{ Primer Name } & \multicolumn{1}{c}{ Primer Sequence (5'to 3') } & $\begin{array}{c}\text { Length of } \\
\text { primer }\end{array}$ & $\begin{array}{c}\text { Product size } \\
\text { (Approx.) }\end{array}$ \\
\hline ProkaryoteV3-Forward & CCTACGGGNBGCASCAG & 17 & \multirow{2}{*}{$460 \mathrm{bps}$} \\
\cline { 1 - 3 } ProkaryoteV4-Reverse & GACTACNVGGGTATCTAATCC & 21 & \\
\hline
\end{tabular}

To generate the cluster as per the standard protocol of Illumina amplicons with the adaptors were amplified by using i5 and i7 primers which add multiplexing index sequences as well as common adapters for the process. The amplicon libraries were then purified by $1 \mathrm{X}$ AM pure XP beads and checked on Agilent High Sensitivity (HS) chipon Bioanalyzer 2100 and quantified for library and mean peak size on fluorometer by Qubit dsDNA HS Assay kit (Life Technologies).

The library was loaded onto Illumina MiSeq platform at appropriate concentration (10-20pM) for cluster generation and sequencing. The kit reagents were used in binding of samples to complementary adapter oligos on paired-end flow cell. The adapters were designed to allow selective cleavage of the forward strands after re-synthesis of the reverse strand during sequencing. The copied reverse strand was then used to sequence from the opposite end of the fragment (Bartram, Lynch, Stearns, Moreno-Hagelsieb, \& Neufeld, 2011).

\section{Data analysis}

Forward and reverse read files were merged using Fast Length Adjustment of Short reads (FLASH) (Magoč \& Salzberg, 2011) to form a single FASTQ file. This file was analysed using Quantitative Insights into Microbial Ecology (QIIME), for microbial community analysis (Caporaso et al., 2010) Operational taxonomical Units (OTUs) were picked based on Uclust_ref method, followed by selection of representative sequences for all OTUs. These representative sequences were aligned using Python Nearest Alignment Space Termination (PyNAST) and Ribosomal Database Project RDP) classifier and
Greengenes version 13.8 databases were used to provide taxonomical identity to OTUs.

\section{RESULTS}

The Illumina MiSeq run of the gut $D$. cingulatus 16 s rRNA amplicon yielded 1 , 10,797 reads. The paired end reads were coupled to generate a single file using the FLASH software. Raw data was submitted to Sequence Read Archive (SRA), NCBI under the Accession ID SRR3355341.

\section{Taxonomic characterization of gut microbes}

We analysed the $16 \mathrm{~S}$ rRNA gene data of gut bacteria of $D$. cingulatus using QIIME and classified as 34 phyla, 88 classes, 132 orders, 200 families, and 345 genera and 171 species. At phylum level, gut bacteria were classified as Proteobacteria (47.10\%), Actinobacteria (25.90\%) and Firmicutes (18\%), (Figure1) (Hassan Salem et al., 2012). Other abundant phyla identified in the gut were Bacteroidetes $(4.20 \%)$, Cyanobacteria (1.40\%), Fusobacteria $(0.30 \%)$, Deferribacteres $(0.20 \%)$, Tenericutes $(0.20 \%)$, Acidobacteria $(0.10 \%)$, and several unclassified bacteria $(0.60 \%)$ (Table 2 \& Figure 1$)$.

Some of the Candidate division bacteria identified in this study were TM7 (Saccharibacteria), GN02 (from Guerrero Negro hyper saline microbial mat), OD1 (Parcubacteria), OP11 (Microgenomates), OP3 (Omnitrophica), SR1 (Abscondita bacteria), WS6 (from Wurtsmith contaminated aquifer), and WWE1 (Cloacimonetes).

Table 2 Percentage of species under phylum level classification

\begin{tabular}{lccccc}
\hline Percentage & Kingdom & Phylum & Percentage & Kingdom & Phylum \\
\hline 47.15 & Bacteria & Proteobacteria & 0.017 & Bacteria & SR1 \\
\hline 25.89 & Bacteria & Actinobacteria & 0.014 & Bacteria & Elusimicrobia \\
\hline 17.96 & Bacteria & Firmicutes & 0.009 & Bacteria & Nitrospirae \\
\hline 4.18 & Bacteria & Bacteroidetes & 0.009 & Bacteria & Synergistetes \\
\hline 1.45 & Bacteria & Cyanobacteria & 0.007 & Bacteria & Armatimonadetes \\
\hline 0.68 & Bacteria & Other & 0.006 & Bacteria & WWE1 \\
\hline 0.29 & Bacteria & Fusobacteria & 0.003 & Bacteria & Chlorobi \\
\hline 0.20 & Bacteria & Tenericutes & 0.003 & Bacteria & WS6 \\
\hline 0.17 & Bacteria & Deferribacteres & 0.002 & Bacteria & BRC1 \\
\hline 0.13 & Bacteria & Acidobacteria & 0.002 & Bacteria & GN02 \\
\hline 0.13 & Bacteria & TM7 & 0.002 & Bacteria & Lentisphaerae \\
\hline 0.12 & Bacteria & Verrucomicrobia & 0.002 & Bacteria & TM6 \\
\hline 0.10 & Bacteria & Chloroflexi & 0.002 & Bacteria & Thermotogae \\
\hline 0.09 & Bacteria & Thermi & 0.002 & Bacteria & WS3 \\
\hline 0.07 & Bacteria & Planctomycetes & 0.001 & Bacteria & Fibrobacteres \\
\hline 0.04 & Bacteria & Gemmatimonadetes & 0.001 & Bacteria & OP11 \\
\hline 0.02 & Bacteria & OD1 & 0.001 & Bacteria & OP3 \\
\hline 0.02 & Bacteria & Spirochaetes & & &
\end{tabular}

At the class level, Proteobacteria consisted of $\gamma$-Proteobacteria $(23.13 \%), \alpha-$ Proteobacteria $(22.36 \%), \beta$-Proteobacteria $(0.77 \%), \delta$-Proteobacteria $(0.37 \%)$, and $\varepsilon$-Proteobacteria $(0.36 \%)$. Actinobacteria consisted of class Actinobacteria $(15.56 \%)$ and Coriobacteria $(10.24 \%)$ and minor classes such as Acidimicrobiia, Thermoleophilia, Rubrobacteria and Nitriliruptoria. Firmicutes were categorized into class Clostridia (10.86\%), Bacilli $(6.91 \%)$, and Erysipelotrichia $(0.068 \%)$ and an unidentified class. Bacteroidetes equals to $4.20 \%$ of total reads and comprised of classes Bacteroidia (3.6\%), Flavobacteria $(0.3 \%)$, Cytophagia $(0.07 \%)$, Sphingobacteria $(0.04 \%)$, and unclassified classes. Similarly, Fusobacteria, Deferribacteres, Tenericutes, comprised of classes Fusobacterium $(0.3 \%)$, Deferribacteres $(0.2 \%)$, and Mollicutes $(0.2 \%)$, respectively (Table $3 \&$ Figure 2).

Most abundant families of Alpha-Proteobacteria were Bartonellaceae (16.5\%), Rhodobacteraceae $(2.2 \%)$, Rhodospirillaceae $(0.43 \%)$ and Rhizobiaceae $(0.1 \%)$. Major families identified in Gamma-Proteobacteria were Enterobacteriaceae (17.6\%), Pseudomonadaceae $(4.4 \%)$, and Xanthomonadaceae $(0.3 \%)$ Coriobacteriaceae $(10.2 \%)$, Bifidobacteriaceae $(8.2 \%)$, Corynebacteriaceae (3.7\%), Micrococcaceae (2\%), Propionibacteriaceae $(0.5 \%)$ and other minor families from the phylum Actinobacteria (Table $4 \&$ Figure 3 ). Gut of $D$. cingulatus also exhibited ample accumulation of Lachnospiraceae (4.8\%),
Tissierellaceae (2.8\%), Streptococcaceae (2.5\%), Bacillaceae (1.5\%), Ruminococcaceae $(0.7 \%)$, Staphylococcaceae $(1.1 \%)$, and Enterococcaceae $(0.2 \%)$ from phylum Firmicutes

Population of the species from the genus Bartonella (Bartonellaceae) was highest with $(17.6 \%)$ of the total diversity in the gut of D. cingulatus. Interestingly, an unidentified genus under the family of Enterobacteriaceae was the next most abundant genus at $1.8 \%$. Gut of D. cingulatus is nourished with genus Klebsiella (3.5\%), Pseudomonas (3.5\%), Paracoccus (2.1\%), Anaerococcus (2.01\%), Erwinia, Acinetobacter, and Enterobacter from Proteobacteria; and Coriobacterium (8.23\%), Bifidobacterium (8.21\%), Corynebacterium (3.9\%), Micrococcus (1.1\%), Rothia, and Propionibacterium from Actinobacteria (Table 5 \& Figure 4).

Alpha diversity calculated for the annotated species was 57.354. Assessment at the base species level, the gut of $D$. cingulatus was rich in Bartonella bacilliformis, Bifidobacterium asteroids, Corynebacterium mucifaciens, Bacillus subtilis Gardnerella vaginalis, Rothia mucilaginosa, and Corynebacterium matruchotii. 


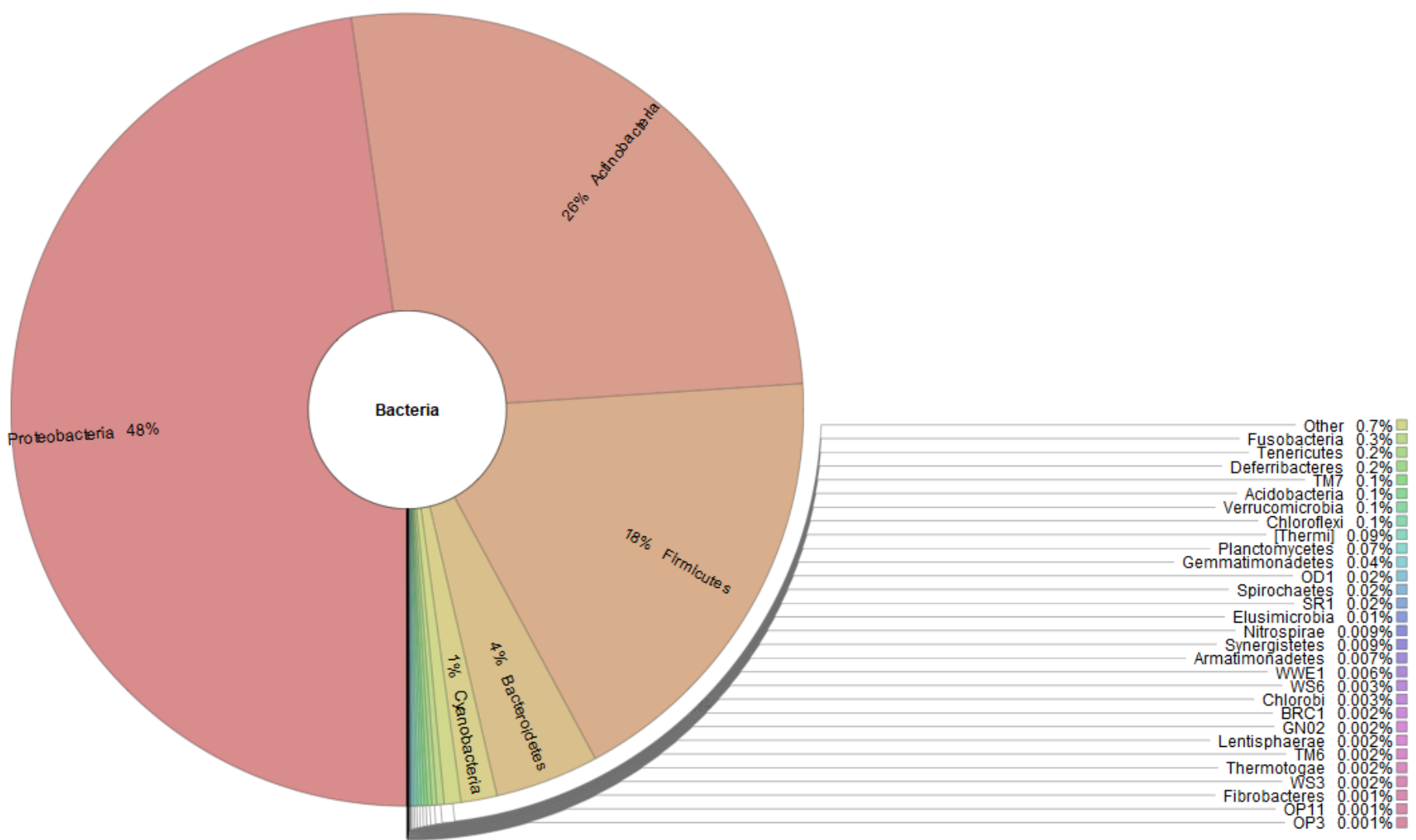

Figure 1 Pie chart showing the distribution of species under phylum level classification

Table 3 Percentage of species under Class level classification

\begin{tabular}{|c|c|c|c|c|c|}
\hline Percentage & Phylum & Class & Percentage & Phylum & Class \\
\hline 23.14 & Proteobacteria & Gammaproteobacteria & 0.01 & Acidobacteria & Sva0725 \\
\hline 22.37 & Proteobacteria & Alphaproteobacteria & 0.01 & Acidobacteria & iii1-8 \\
\hline 15.57 & Actinobacteria & Actinobacteria & 0.01 & Actinobacteria & Other \\
\hline 10.86 & Firmicutes & Clostridia & 0.01 & Chloroflexi & TK10 \\
\hline 10.24 & Actinobacteria & Coriobacteriia & 0.01 & Chloroflexi & Other \\
\hline 6.91 & Firmicutes & Bacilli & 0.01 & Cyanobacteria & Oscillatoriophycideae \\
\hline 3.60 & Bacteroidetes & Bacteroidia & 0.01 & Gemmatimonadetes & Gemm-5 \\
\hline 1.41 & Cyanobacteria & Chloroplast & 0.01 & $W W E 1$ & Cloacamonae \\
\hline 0.78 & Proteobacteria & Betaproteobacteria & 0.01 & Actinobacteria & MB-A2-108 \\
\hline 0.68 & Other & Other & 0.01 & $O D 1$ & - \\
\hline 0.37 & Proteobacteria & Deltaproteobacteria & 0.01 & $T M 7$ & TM7-1 \\
\hline 0.36 & Proteobacteria & Epsilonproteobacteria & 0.0041 & Acidobacteria & Acidobacteriia \\
\hline 0.31 & Bacteroidetes & Flavobacteriia & 0.0041 & Acidobacteria & TM1 \\
\hline 0.29 & Fusobacteria & Fusobacteriia & 0.0041 & Verrucomicrobia & Other \\
\hline 0.20 & Tenericutes & Mollicutes & 0.0031 & Armatimonadetes & 0319-6Е2 \\
\hline 0.17 & Deferribacteres & Deferribacteres & 0.0031 & Bacteroidetes & Rhodothermi \\
\hline 0.13 & Proteobacteria & Other & 0.0031 & Chloroflexi & TK17 \\
\hline 0.13 & Bacteroidetes & Other & 0.0031 & Cyanobacteria & - \\
\hline 0.12 & Firmicutes & Other & 0.0031 & Spirochaetes & Brachyspirae \\
\hline 0.12 & $T M 7$ & TM7-3 & 0.0031 & WS6 & $\mathrm{SC72}$ \\
\hline 0.09 & Thermi & Deinococci & 0.0021 & Acidobacteria & S035 \\
\hline 0.07 & Bacteroidetes & Cytophagia & 0.0021 & Armatimonadetes & Chthonomonadetes \\
\hline 0.07 & Verrucomicrobia & Opitutae & 0.0021 & $B R C 1$ & PRR-11 \\
\hline 0.07 & Firmicutes & Erysipelotrichi & 0.0021 & Chloroflexi & Ktedonobacteria \\
\hline 0.05 & Acidobacteria & Acidobacteria-6 & 0.0021 & Gemmatimonadetes & - \\
\hline 0.04 & Planctomycetes & Planctomycetia & 0.0021 & Lentisphaerae & Lentisphaeria \\
\hline 0.04 & Bacteroidetes & Sphingobacteriia & 0.0021 & Planctomycetes & OM190 \\
\hline 0.04 & Chloroflexi & Thermomicrobia & 0.0021 & TM6 & SJA-4 \\
\hline 0.04 & Actinobacteria & Acidimicrobiia & 0.0021 & $T M 7$ & - \\
\hline 0.03 & Bacteroidetes & Saprospirae & 0.0021 & Thermotogae & Thermotogae \\
\hline 0.03 & Actinobacteria & Thermoleophilia & 0.0021 & WS3 & PRR-12 \\
\hline 0.02 & Chloroflexi & Anaerolineae & 0.0010 & Acidobacteria & Other \\
\hline
\end{tabular}




\begin{tabular}{llllll}
\hline 0.02 & Planctomycetes & Phycisphaerae & 0.0010 & Acidobacteria & AT-s54 \\
\hline 0.02 & Acidobacteria & Chloracidobacteria & 0.0010 & Acidobacteria & Acidobacteria-5 \\
\hline 0.02 & Acidobacteria & Solibacteres & 0.0010 & Acidobacteria & EC1113 \\
\hline 0.02 & Spirochaetes & Spirochaetes & 0.0010 & Actinobacteria & Nitriliruptoria \\
\hline 0.02 & Verrucomicrobia & Spartobacteria & 0.0010 & Armatimonadetes & Armatimonadia \\
\hline 0.02 & Gemmatimonadetes & Gemm-3 & 0.0010 & Armatimonadetes & Fimbriimonadia \\
\hline 0.02 & Gemmatimonadetes & Gemmatimonadetes & 0.0010 & Chlorobi & - \\
\hline 0.02 & SR1 & - & 0.0010 & Chlorobi & OPB56 \\
\hline 0.02 & Cyanobacteria & Other & 0.0010 & Chlorobi & SJA-28 \\
\hline 0.02 & Cyanobacteria & 4C0d-2 & 0.0010 & Chloroflexi & SAR202 \\
\hline 0.02 & OD1 & ZB2 & 0.0010 & Fibrobacteres & Fibrobacteria \\
\hline 0.02 & Verrucomicrobia & Pedosphaerae & 0.0010 & GN02 & BD1-5 \\
\hline 0.01 & Elusimicrobia & Elusimicrobia & 0.0010 & GN02 & GKS2-174 \\
\hline 0.01 & Verrucomicrobia & Verrucomicrobiae & 0.0010 & OD1 & ABY1 \\
\hline 0.01 & Actinobacteria & Rubrobacteria & 0.0010 & OD1 & SM2F11 \\
\hline 0.01 & Acidobacteria & BPC102 & 0.0010 & OP11 & WCHB1-64 \\
\hline 0.01 & Chloroflexi & Chloroflexi & 0.0010 & OP3 & koll11 \\
\hline 0.01 & Nitrospirae & Nitrospira & 0.0010 & Planctomycetes & vadinHA49 \\
\hline 0.01 & Synergistetes & Synergistia & 0.0010 & Proteobacteria & TA18 \\
\hline 0.01 & Chloroflexi & Ellin6529 & 0.0010 & TM7 & Other \\
\hline
\end{tabular}

Legend: '-' Classification data not available in the database.

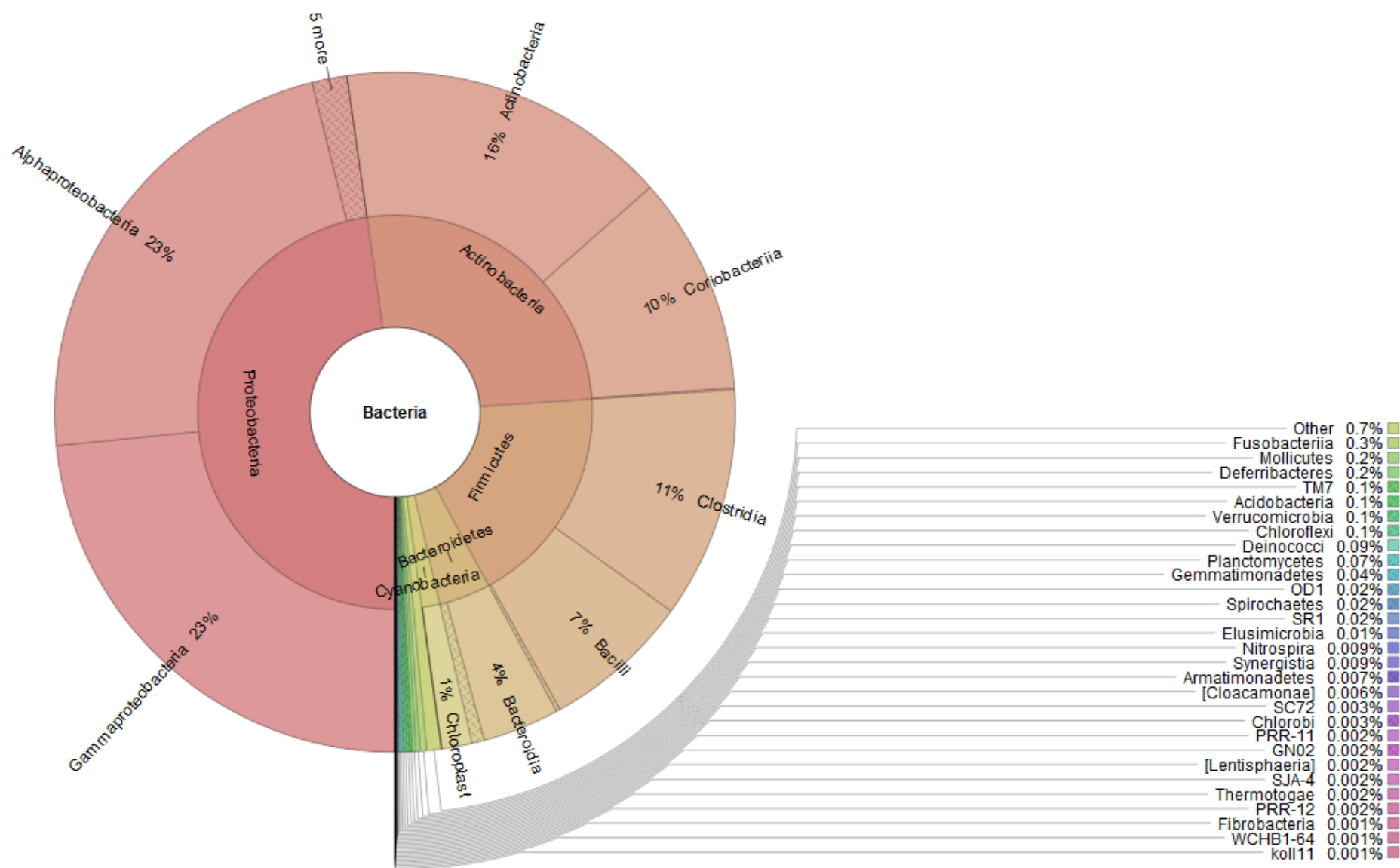

Figure 2 Pie chart showing the distribution of species under Class level classification

Table 4 Percentage of species under Family level classification

\begin{tabular}{cllcll}
\hline Percentage & \multicolumn{1}{c}{ Order } & \multicolumn{1}{c}{ Family } & Percentage & \multicolumn{1}{c}{ Order } & \multicolumn{1}{c}{ Family } \\
\hline 17.65 & Enterobacteriales & Enterobacteriaceae & 0.010 & Rickettsiales & - \\
\hline 16.48 & Rhizobiales & Bartonellaceae & 0.010 & Bdellovibrionales & Bdellovibrionaceae \\
\hline 10.24 & Coriobacteriales & Coriobacteriaceae & 0.009 & iiil-15 & mb2424 \\
\hline 8.23 & Bifidobacteriales & Bifidobacteriaceae & 0.009 & Gaiellales & Gaiellaceae \\
\hline 4.77 & Clostridiales & Lachnospiraceae & 0.009 & Clostridiales & Acidaminobacteraceae \\
\hline 3.71 & Actinomycetales & Corynebacteriaceae & 0.008 & Actinomycetales & Pseudonocardiaceae \\
\hline 3.30 & Pseudomonadales & Pseudomonadaceae & 0.008 & Cytophagales & Flammeovirgaceae \\
\hline 2.76 & Clostridiales & Tissierellaceae & 0.008 & Elusimicrobiales & Elusimicrobiaceae \\
\hline 2.50 & Lactobacillales & Streptococcaceae & 0.008 & Nitrospirales & 0319-6A21 \\
\hline
\end{tabular}




\begin{tabular}{|c|c|c|c|c|c|}
\hline 2.25 & Rhodobacterales & Rhodobacteraceae & 0.008 & Gemmatales & Isosphaeraceae \\
\hline 2.04 & Actinomycetales & Micrococcaceae & 0.007 & Acidimicrobiales & $C 111$ \\
\hline 1.47 & Bacillales & Bacillaceae & 0.007 & Actinomycetales & Geodermatophilaceae \\
\hline 1.10 & Clostridiales & Ruminococcaceae & 0.007 & Actinomycetales & Micromonosporaceae \\
\hline 1.09 & Bacillales & Staphylococcaceae & 0.007 & Flavobacteriales & Cryomorphaceae \\
\hline 1.09 & Pseudomonadales & Moraxellaceae & 0.007 & Rhizobiales & Beijerinckiaceae \\
\hline 0.91 & Bacteroidales & Prevotellaceae & 0.007 & Rhizobiales & Xanthobacteraceae \\
\hline 0.67 & Bacteroidales & $S 24-7$ & 0.007 & Syntrophobacterales & Syntrophobacteraceae \\
\hline 0.49 & Actinomycetales & Propionibacteriaceae & 0.006 & Solirubrobacterales & Solirubrobacteraceae \\
\hline 0.44 & Clostridiales & Veillonellaceae & 0.006 & Caldilineales & Caldilineaceae \\
\hline 0.43 & Rhodospirillales & Rhodospirillaceae & 0.006 & Gemmatales & Gemmataceae \\
\hline 0.40 & Rickettsiales & mitochondria & 0.006 & Planctomycetales & Planctomycetaceae \\
\hline 0.40 & Neisseriales & Neisseriaceae & 0.006 & Rickettsiales & Rickettsiaceae \\
\hline 0.34 & Bacteroidales & Rikenellaceae & 0.006 & Rhodocyclales & Rhodocyclaceae \\
\hline 0.34 & Bacteroidales & Porphyromonadaceae & 0.005 & Actinomycetales & Dermatophilaceae \\
\hline 0.34 & Bacteroidales & Paraprevotellaceae & 0.005 & Actinomycetales & Nocardiaceae \\
\hline 0.33 & Bacteroidales & Bacteroidaceae & 0.005 & Bacteroidales & Barnesiellaceae \\
\hline 0.32 & Campylobacterales & Helicobacteraceae & 0.005 & Cytophagales & Cyclobacteriaceae \\
\hline 0.31 & Bacteroidales & Odoribacteraceae & 0.005 & Oscillatoriales & Phormidiaceae \\
\hline 0.31 & Xanthomonadales & Xanthomonadaceae & 0.005 & Vibrionales & Pseudoalteromonadaceae \\
\hline 0.29 & Desulfovibrionales & Desulfovibrionaceae & 0.004 & Acidobacteriales & Acidobacteriaceae \\
\hline 0.27 & Lactobacillales & Aerococcaceae & 0.004 & Solibacterales & Solibacteraceae \\
\hline 0.23 & Lactobacillales & Leuconostocaceae & 0.004 & Actinomycetales & Dermacoccaceae \\
\hline 0.22 & Lactobacillales & Enterococcaceae & 0.004 & Bacteroidales & BA008 \\
\hline 0.22 & Flavobacteriales & Flavobacteriaceae & 0.004 & $A K Y G 885$ & Dolo_23 \\
\hline 0.19 & Fusobacteriales & Leptotrichiaceae & 0.004 & Bacillales & Alicyclobacillaceae \\
\hline 0.19 & Rhizobiales & Aurantimonadaceae & 0.004 & Rhodobacterales & Hyphomonadaceae \\
\hline 0.19 & Vibrionales & Vibrionaceae & 0.004 & Oceanospirillales & Oceanospirillaceae \\
\hline 0.18 & Gemellales & Gemellaceae & 0.004 & Salinisphaerales & Salinisphaeraceae \\
\hline 0.17 & Deferribacterales & Deferribacteraceae & 0.004 & Synergistales & Dethiosulfovibrionaceae \\
\hline 0.17 & Lactobacillales & Carnobacteriaceae & 0.004 & Pedosphaerales & $R 4-41 B$ \\
\hline 0.16 & Pasteurellales & Pasteurellaceae & 0.003 & Acidimicrobiales & AKIW874 \\
\hline 0.15 & Mycoplasmatales & Mycoplasmataceae & 0.003 & Actinomycetales & Glycomycetaceae \\
\hline 0.14 & Rhodospirillales & Acetobacteraceae & 0.003 & Actinomycetales & Sporichthyaceae \\
\hline 0.14 & Sphingomonadales & Sphingomonadaceae & 0.003 & Solirubrobacterales & Patulibacteraceae \\
\hline 0.12 & Burkholderiales & Comamonadaceae & 0.003 & Rhodothermales & Balneolaceae \\
\hline 0.11 & Actinomycetales & Intrasporangiaceae & 0.003 & Anaerolineales & Anaerolinaceae \\
\hline 0.11 & Rhizobiales & Rhizobiaceae & 0.003 & Clostridiales & Christensenellaceae \\
\hline 0.10 & Fusobacteriales & Fusobacteriaceae & 0.003 & Desulfovibrionales & Desulfomicrobiaceae \\
\hline 0.10 & Actinomycetales & Actinomycetaceae & 0.003 & Myxococcales & Nannocystaceae \\
\hline 0.09 & Lactobacillales & Lactobacillaceae & 0.003 & Syntrophobacterales & Syntrophaceae \\
\hline 0.09 & Actinomycetales & Dietziaceae & 0.003 & Alteromonadales & $211 d s 20$ \\
\hline 0.09 & Clostridiales & Mogibacteriaceae & 0.003 & Thiotrichales & Piscirickettsiaceae \\
\hline 0.08 & Actinomycetales & Nocardioidaceae & 0.003 & Brachyspirales & Brachyspiraceae \\
\hline 0.08 & Actinomycetales & Dermabacteraceae & 0.003 & Pedosphaerales & Ellin517 \\
\hline 0.07 & Methylophilales & Methylophilaceae & 0.003 & Cloacamonales & $C W-1$ \\
\hline 0.07 & Rhizobiales & Brucellaceae & 0.003 & Cloacamonales & Cloacamonaceae \\
\hline 0.07 & Actinomycetales & Microbacteriaceae & 0.002 & Acidimicrobiales & koll13 \\
\hline 0.07 & Flavobacteriales & Weeksellaceae & 0.002 & Actinomycetales & Kineosporiaceae \\
\hline 0.07 & Erysipelotrichales & Erysipelotrichaceae & 0.002 & Actinomycetales & Mycobacteriaceae \\
\hline 0.07 & Clostridiales & Clostridiaceae & 0.002 & Actinomycetales & Promicromonosporaceae \\
\hline 0.07 & Alteromonadales & Chromatiaceae & 0.002 & Gaiellales & AK1AB1_O2E \\
\hline 0.07 & Rhizobiales & Methylobacteriaceae & 0.002 & Bacteroidales & $S B-1$ \\
\hline 0.06 & Bacillales & Planococcaceae & 0.002 & Turicibacterales & Turicibacteraceae \\
\hline 0.06 & Thermales & Thermaceae & 0.002 & Gemmatimonadales & Gemmatimonadaceae \\
\hline 0.06 & Actinomycetales & Streptomycetaceae & 0.002 & $\mathrm{Z} 20$ & $R 4-45 B$ \\
\hline 0.06 & Aeromonadales & Aeromonadaceae & 0.002 & Rhizobiales & Rhodobiaceae \\
\hline 0.05 & Burkholderiales & Burkholderiaceae & 0.002 & Nitrosomonadales & Nitrosomonadaceae \\
\hline 0.05 & Burkholderiales & Oxalobacteraceae & 0.002 & Desulfarculales & Desulfarculaceae \\
\hline 0.05 & Caulobacterales & Caulobacteraceae & 0.002 & Myxococcales & Cystobacteraceae \\
\hline 0.04 & Rhizobiales & Bradyrhizobiaceae & 0.002 & Myxococcales & Myxococcaceae \\
\hline 0.04 & Anaeroplasmatales & Anaeroplasmataceae & 0.002 & Oceanospirillales & Hahellaceae \\
\hline
\end{tabular}




\begin{tabular}{|c|c|c|c|c|c|}
\hline 0.04 & Sphingomonadales & Erythrobacteraceae & 0.002 & Sphaerochaetales & Sphaerochaetaceae \\
\hline 0.04 & Campylobacterales & Campylobacteraceae & 0.002 & Thermotogales & Thermotogaceae \\
\hline 0.04 & Cytophagales & Cytophagaceae & 0.002 & Deinococcales & Trueperaceae \\
\hline 0.04 & Sphingobacteriales & Sphingobacteriaceae & 0.001 & Solibacterales & Bryobacteraceae \\
\hline 0.04 & Clostridiales & Peptostreptococcaceae & 0.001 & Acidimicrobiales & EB1017 \\
\hline 0.04 & Actinomycetales & Brevibacteriaceae & 0.001 & Actinomycetales & Actinopolysporaceae \\
\hline 0.04 & Bacillales & Exiguobacteraceae & 0.001 & Actinomycetales & Actinosynnemataceae \\
\hline 0.03 & Alteromonadales & Alteromonadaceae & 0.001 & Actinomycetales & Bogoriellaceae \\
\hline 0.03 & Rhizobiales & Hyphomicrobiaceae & 0.001 & Actinomycetales & Thermomonosporaceae \\
\hline 0.03 & Burkholderiales & Alcaligenaceae & 0.001 & Actinomycetales & Yaniellaceae \\
\hline 0.03 & Saprospirales & Chitinophagaceae & 0.001 & Nitriliruptorales & Nitriliruptoraceae \\
\hline 0.03 & Deinococcales & Deinococcaceae & 0.001 & Armatimonadales & Armatimonadaceae \\
\hline 0.02 & Oceanospirillales & Halomonadaceae & 0.001 & Chthonomonadales & Chthonomonadaceae \\
\hline 0.02 & Rhizobiales & Phyllobacteriaceae & 0.001 & Fimbriimonadales & Fimbriimonadaceae \\
\hline 0.02 & Pirellulales & Pirellulaceae & 0.001 & Saprospirales & Saprospiraceae \\
\hline 0.02 & Clostridiales & Peptococcaceae & 0.001 & Ardenscatenales & Ardenscatenaceae \\
\hline 0.02 & Alteromonadales & Shewanellaceae & 0.001 & Thermogemmatisporales & Thermogemmatisporaceae \\
\hline 0.02 & Chthoniobacterales & Chthoniobacteraceae & 0.001 & Clostridiales & $\mathrm{EtOH} 8$ \\
\hline 0.02 & Bacillales & Paenibacillaceae & 0.001 & Nitrospirales & Nitrospiraceae \\
\hline 0.02 & Xanthomonadales & Sinobacteraceae & 0.001 & Phycisphaerales & Phycisphaeraceae \\
\hline 0.02 & Spirochaetales & Spirochaetaceae & 0.001 & Kiloniellales & Kiloniellaceae \\
\hline 0.01 & Legionellales & Legionellaceae & 0.001 & Rhizobiales & Methylocystaceae \\
\hline 0.01 & Verrucomicrobiales & Verrucomicrobiaceae & 0.001 & Myxococcales & Polyangiaceae \\
\hline 0.01 & $R B 41$ & Ellin6075 & 0.001 & $N B 1-j$ & $N B 1-i$ \\
\hline 0.01 & Rubrobacterales & Rubrobacteraceae & 0.001 & Alteromonadales & HTCC2188 \\
\hline 0.01 & Legionellales & Coxiellaceae & 0.001 & Alteromonadales & Idiomarinaceae \\
\hline 0.01 & Opitutales & Opitutaceae & 0.001 & Cardiobacteriales & Cardiobacteriaceae \\
\hline 0.01 & Actinomycetales & Cellulomonadaceae & 0.001 & 1025 & $R s-045$ \\
\hline 0.01 & Actinomycetales & Gordoniaceae & 0.001 & Pedosphaerales & Ellin515 \\
\hline
\end{tabular}

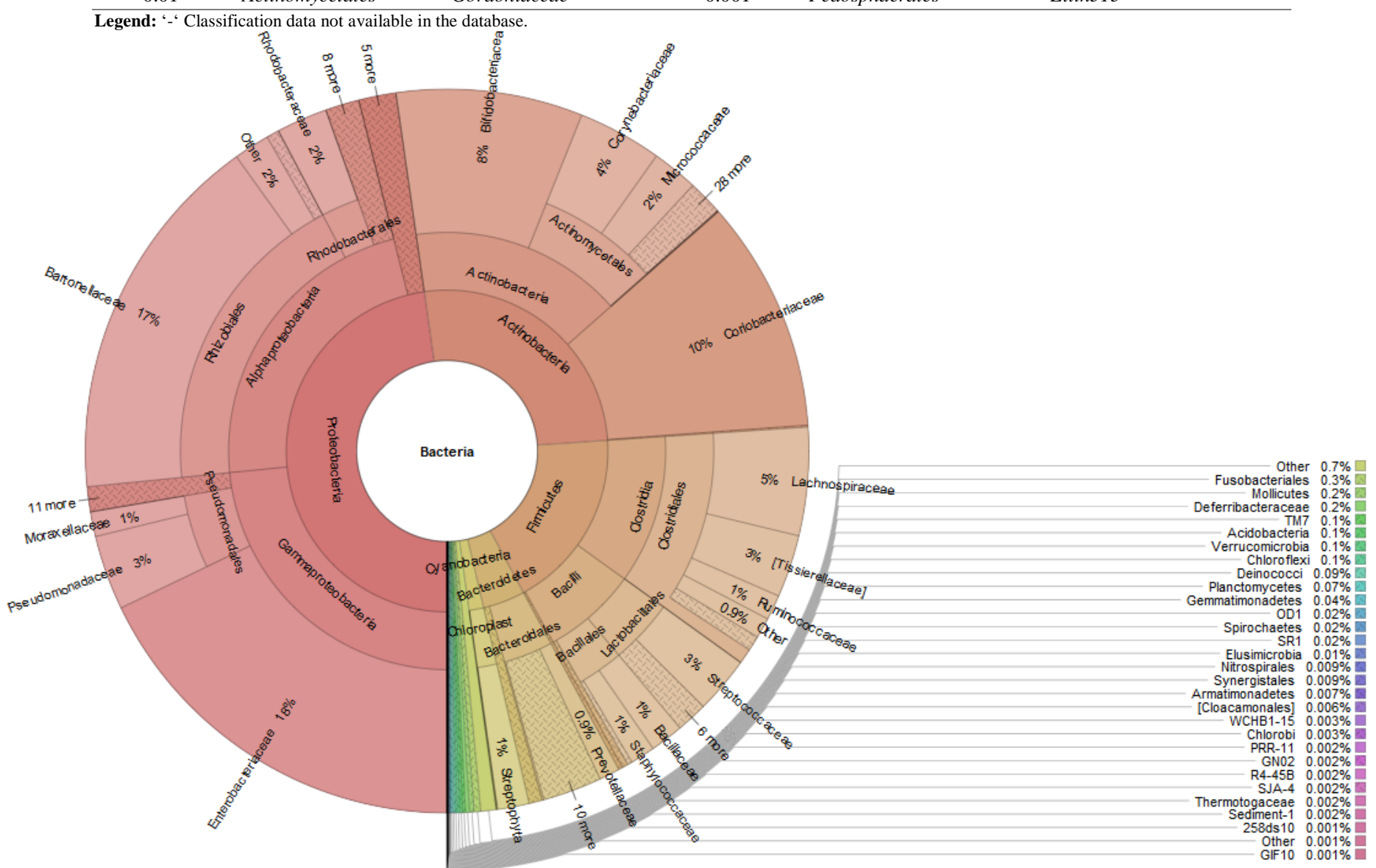

Figure 3 Pie chart showing the distribution of bacteria at Family level classification 
Table 5 Percentage of species under phylum Genus classification

\begin{tabular}{|c|c|c|c|c|c|}
\hline Percentage & Family & Genus & Percentage & Family & Genus \\
\hline 8.305552 & Coriobacteriaceae & Coriobacterium & 0.006212 & Alteromonadaceae & Marinobacter \\
\hline 3.734496 & Bifidobacteriaceae & Bifidobacterium & 0.005177 & Dermatophilaceae & Piscicoccus \\
\hline 3.708612 & Corynebacteriaceae & Corynebacterium & 0.005177 & Nocardiaceae & Rhodococcus \\
\hline 3.004576 & Pseudomonadaceae & Pseudomonas & 0.005177 & Bacillaceae & Lentibacillus \\
\hline 2.49415 & Streptococcaceae & Streptococcus & 0.005177 & Peptostreptococcaceae & Filifactor \\
\hline 2.094506 & Rhodobacteraceae & Paracoccus & 0.005177 & Veillonellaceae & Mitsuokella \\
\hline 1.977512 & Tissierellaceae & Anaerococcus & 0.005177 & Alcaligenaceae & Achromobacter \\
\hline 1.29936 & Bacillaceae & Bacillus & 0.005177 & Alcaligenaceae & Sutterella \\
\hline 1.16373 & Lachnospiraceae & Clostridium & 0.005177 & Neisseriaceae & Vogesella \\
\hline 1.018781 & Micrococcaceae & Micrococcus & 0.005177 & Desulfovibrionaceae & Bilophila \\
\hline 0.909034 & Prevotellaceae & Prevotella & 0.005177 & Moraxellaceae & Perlucidibaca \\
\hline 0.8055 & Moraxellaceae & Acinetobacter & 0.005177 & Verrucomicrobiaceae & Prosthecobacter \\
\hline 0.803429 & Staphylococcaceae & Staphylococcus & 0.004141 & Dermacoccaceae & Dermacoccus \\
\hline 0.680223 & Micrococcaceae & Rothia & 0.004141 & Microbacteriaceae & Pseudoclavibacter \\
\hline 0.680223 & Ruminococcaceae & Oscillospira & 0.004141 & Micrococcaceae & Nesterenkonia \\
\hline 0.658481 & Enterobacteriaceae & Citrobacter & 0.004141 & Nocardioidaceae & Aeromicrobium \\
\hline 0.473153 & Propionibacteriaceae & Propionibacterium & 0.004141 & Coriobacteriaceae & Eggerthella \\
\hline 0.399644 & Tissierellaceae & $G W-34$ & 0.004141 & Bacteroidaceae & $5-7 N 15$ \\
\hline 0.323028 & Bacteroidaceae & Bacteroides & 0.004141 & Porphyromonadaceae & Tannerella \\
\hline 0.310604 & Odoribacteraceae & Odoribacter & 0.004141 & Cytophagaceae & Rhodocytophaga \\
\hline 0.29818 & Neisseriaceae & Neisseria & 0.004141 & Phormidiaceae & Phormidium \\
\hline 0.272296 & Paraprevotellaceae & Prevotella & 0.004141 & Alicyclobacillaceae & Alicyclobacillus \\
\hline 0.261943 & Rhodospirillaceae & Rhodospirillum & 0.004141 & Bacillaceae & Marinibacillus \\
\hline 0.235024 & Porphyromonadaceae & Porphyromonas & 0.004141 & Planococcaceae & Planomicrobium \\
\hline 0.218458 & Leuconostocaceae & Weissella & 0.004141 & Planococcaceae & Rummeliibacillus \\
\hline 0.218458 & Xanthomonadaceae & Stenotrophomonas & 0.004141 & Enterococcaceae & Enterococcus \\
\hline 0.197751 & Ruminococcaceae & Ruminococcus & 0.004141 & Ruminococcaceae & Faecalibacterium \\
\hline 0.184292 & Helicobacteraceae & Helicobacter & 0.004141 & Aurantimonadaceae & Aurantimonas \\
\hline 0.174974 & Vibrionaceae & Photobacterium & 0.004141 & Phyllobacteriaceae & Chelativorans \\
\hline 0.172903 & Micrococcaceae & Kocuria & 0.004141 & Acetobacteraceae & Roseococcus \\
\hline 0.169797 & Deferribacteraceae & Mucispirillum & 0.004141 & Acetobacteraceae & Roseomonas \\
\hline 0.160479 & Moraxellaceae & Enhydrobacter & 0.004141 & Comamonadaceae & Limnohabitans \\
\hline 0.154267 & Leptotrichiaceae & Leptotrichia & 0.004141 & Salinisphaeraceae & Salinisphaera \\
\hline 0.145984 & Enterobacteriaceae & Escherichia & 0.004141 & Pseudoalteromonadaceae & Vibrio \\
\hline 0.128383 & Tissierellaceae & Peptoniphilus & 0.004141 & Xanthomonadaceae & Xanthomonas \\
\hline 0.127348 & Veillonellaceae & Veillonella & 0.004141 & Dethiosulfovibrionaceae & Pyramidobacter \\
\hline 0.122171 & Aerococcaceae & Alloiococcus & 0.003106 & Actinomycetaceae & Arcanobacterium \\
\hline 0.094217 & Actinomycetaceae & Actinomyces & 0.003106 & Cellulomonadaceae & Cellulomonas \\
\hline 0.084898 & Flavobacteriaceae & Capnocytophaga & 0.003106 & Geodermatophilaceae & Geodermatophilus \\
\hline 0.081792 & Porphyromonadaceae & Parabacteroides & 0.003106 & Glycomycetaceae & Glycomyces \\
\hline 0.079722 & Fusobacteriaceae & Fusobacterium & 0.003106 & Microbacteriaceae & Candidatus Aquiluna \\
\hline 0.076616 & Pasteurellaceae & Haemophilus & 0.003106 & Patulibacteraceae & Patulibacter \\
\hline 0.071439 & Brucellaceae & Ochrobactrum & 0.003106 & Odoribacteraceae & Butyricimonas \\
\hline 0.069368 & Dermabacteraceae & Brachybacterium & 0.003106 & Cytophagaceae & Leadbetterella \\
\hline 0.067298 & Tissierellaceae & $W A L \_1855 D$ & 0.003106 & Flavobacteriaceae & Gillisia \\
\hline 0.065227 & Methylophilaceae & Methylotenera & 0.003106 & Anaerolinaceae & Anaerolinea \\
\hline 0.062121 & Tissierellaceae & $1-64$ & 0.003106 & Planococcaceae & Lysinibacillus \\
\hline 0.062121 & Moraxellaceae & Moraxella & 0.003106 & Carnobacteriaceae & Desemzia \\
\hline 0.06005 & Dietziaceae & Dietzia & 0.003106 & Streptococcaceae & Lactococcus \\
\hline 0.059015 & Sphingomonadaceae & Sphingomonas & 0.003106 & Mogibacteriaceae & Mogibacterium \\
\hline 0.059015 & Helicobacteraceae & Flexispira & 0.003106 & Erysipelotrichaceae & Asteroleplasma \\
\hline 0.057979 & Streptomycetaceae & Streptomyces & 0.003106 & Fusobacteriaceae & Cetobacterium \\
\hline 0.056944 & Aerococcaceae & Aerococcus & 0.003106 & Hyphomicrobiaceae & Rhodoplanes \\
\hline 0.054873 & Paraprevotellaceae & $Y R C 22$ & 0.003106 & Oxalobacteraceae & Massilia \\
\hline 0.051767 & Clostridiaceae & Clostridium & 0.003106 & Neisseriaceae & Conchiformibius \\
\hline 0.051767 & Erysipelotrichaceae & Bulleidia & 0.003106 & Rhodocyclaceae & Dechloromonas \\
\hline 0.051767 & Desulfovibrionaceae & Desulfovibrio & 0.003106 & Desulfomicrobiaceae & Desulfomicrobium \\
\hline 0.049697 & Intrasporangiaceae & Serinicoccus & 0.003106 & Enterobacteriaceae & Erwinia \\
\hline 0.047626 & Rikenellaceae & Alistipes & 0.003106 & Oceanospirillaceae & Oleibacter \\
\hline 0.047626 & Tissierellaceae & Parvimonas & 0.003106 & Moraxellaceae & Alkanindiges \\
\hline 0.045555 & Aerococcaceae & Facklamia & 0.003106 & Xanthomonadaceae & Thermomonas \\
\hline
\end{tabular}




\begin{tabular}{|c|c|c|c|c|c|}
\hline 0.04452 & Rhodospirillaceae & Azospirillum & 0.003106 & Brachyspiraceae & Brachyspira \\
\hline 0.043485 & Lactobacillaceae & Lactobacillus & 0.003106 & Mycoplasmataceae & Mycoplasma \\
\hline 0.043485 & Methylobacteriaceae & Methylobacterium & 0.003106 & Chthoniobacteraceae & heteroC45_4W \\
\hline 0.042449 & Tissierellaceae & $p h 2$ & 0.003106 & Cloacamonaceae & $W 22$ \\
\hline 0.041414 & Lachnospiraceae & Oribacterium & 0.002071 & Solibacteraceae & Candidatus Solibacter \\
\hline 0.041414 & mitochondria & Lupinus & 0.002071 & Dermabacteraceae & Dermabacter \\
\hline 0.041414 & Anaeroplasmataceae & Anaeroplasma & 0.002071 & Microbacteriaceae & Clavibacter \\
\hline 0.041414 & Thermaceae & Thermus & 0.002071 & Mycobacteriaceae & Mycobacterium \\
\hline 0.036237 & Brevibacteriaceae & Brevibacterium & 0.002071 & Promicromonosporaceae & Promicromonospora \\
\hline 0.036237 & Staphylococcaceae & Jeotgalicoccus & 0.002071 & Propionibacteriaceae & Brooklawnia \\
\hline 0.034166 & Staphylococcaceae & Macrococcus & 0.002071 & Propionibacteriaceae & Tessaracoccus \\
\hline 0.034166 & Veillonellaceae & Dialister & 0.002071 & Pseudonocardiaceae & Actinomycetospora \\
\hline 0.034166 & Burkholderiaceae & Burkholderia & 0.002071 & Bifidobacteriaceae & Alloscardovia \\
\hline 0.033131 & Lachnospiraceae & Ruminococcus & 0.002071 & Coriobacteriaceae & Collinsella \\
\hline 0.032096 & Sphingomonadaceae & Novosphingobium & 0.002071 & Coriobacteriaceae & Slackia \\
\hline 0.032096 & Enterobacteriaceae & Klebsiella & 0.002071 & Porphyromonadaceae & Dysgonomonas \\
\hline 0.03106 & Micrococcaceae & Microbispora & 0.002071 & Cyclobacteriaceae & Algoriphagus \\
\hline 0.03106 & Chromatiaceae & Rheinheimera & 0.002071 & Cytophagaceae & Adhaeribacter \\
\hline 0.03106 & Pasteurellaceae & Actinobacillus & 0.002071 & Weeksellaceae & Cloacibacterium \\
\hline 0.030025 & Staphylococcaceae & Salinicoccus & 0.002071 & Bacillaceae & Virgibacillus \\
\hline 0.030025 & Campylobacteraceae & Campylobacter & 0.002071 & Turicibacteraceae & Turicibacter \\
\hline 0.02899 & Deinococcaceae & Deinococcus & 0.002071 & Lachnospiraceae & Butyrivibrio \\
\hline 0.027954 & Veillonellaceae & Megasphaera & 0.002071 & Lachnospiraceae & Defluviitalea \\
\hline 0.025884 & Veillonellaceae & Selenomonas & 0.002071 & Lachnospiraceae & Dorea \\
\hline 0.025884 & Enterobacteriaceae & Serratia & 0.002071 & Ruminococcaceae & Clostridium \\
\hline 0.024848 & Coriobacteriaceae & Atopobium & 0.002071 & Veillonellaceae & Schwartzia \\
\hline 0.024848 & Peptostreptococcaceae & Peptostreptococcus & 0.002071 & Erysipelotrichaceae & Catenibacterium \\
\hline 0.023813 & Flavobacteriaceae & Flavobacterium & 0.002071 & Erysipelotrichaceae & RFN20 \\
\hline 0.023813 & Hyphomicrobiaceae & Devosia & 0.002071 & Gemmatimonadaceae & Gemmatimonas \\
\hline 0.022778 & Caulobacteraceae & Brevundimonas & 0.002071 & Gemmataceae & Gemmata \\
\hline 0.022778 & Rhodospirillaceae & Skermanella & 0.002071 & Pirellulaceae & $A 17$ \\
\hline 0.020707 & Microbacteriaceae & Microbacterium & 0.002071 & Caulobacteraceae & Caulobacter \\
\hline 0.020707 & Weeksellaceae & Chryseobacterium & 0.002071 & Caulobacteraceae & Mycoplana \\
\hline 0.019672 & Shewanellaceae & Shewanella & 0.002071 & Rhizobiaceae & Rhizobium \\
\hline 0.019672 & Pasteurellaceae & Aggregatibacter & 0.002071 & Rhodospirillaceae & Inquilinus \\
\hline 0.018636 & Bradyrhizobiaceae & Balneimonas & 0.002071 & Neisseriaceae & Eikenella \\
\hline 0.018636 & Chromatiaceae & Alishewanella & 0.002071 & Nitrosomonadaceae & Nitrosovibrio \\
\hline 0.017601 & Cytophagaceae & Pontibacter & 0.002071 & Rhodocyclaceae & C39 \\
\hline 0.017601 & Sphingobacteriaceae & Sphingobacterium & 0.002071 & Myxococcaceae & Myxococcus \\
\hline 0.017601 & Lachnospiraceae & Coprococcus & 0.002071 & Nannocystaceae & Nannocystis \\
\hline 0.016566 & Chitinophagaceae & Flavisolibacter & 0.002071 & Alteromonadaceae & Microbulbifer \\
\hline 0.016566 & Peptococcaceae & Peptococcus & 0.002071 & Enterobacteriaceae & Proteus \\
\hline 0.016566 & Rhizobiaceae & Shinella & 0.002071 & Coxiellaceae & Aquicella \\
\hline 0.016566 & Rhodobacteraceae & Rhodobacter & 0.002071 & Coxiellaceae & Rickettsiella \\
\hline 0.016566 & Rhodospirillaceae & Novispirillum & 0.002071 & Hahellaceae & Hahella \\
\hline 0.016566 & Alteromonadaceae & Cellvibrio & 0.002071 & Xanthomonadaceae & Dokdonella \\
\hline 0.016566 & Moraxellaceae & Psychrobacter & 0.002071 & Xanthomonadaceae & Rhodanobacter \\
\hline 0.016566 & Thermaceae & Meiothermus & 0.002071 & Sphaerochaetaceae & Sphaerochaeta \\
\hline 0.01553 & Carnobacteriaceae & Granulicatella & 0.002071 & Thermotogaceae & AUTHM297 \\
\hline 0.01553 & Sphingomonadaceae & Kaistobacter & 0.002071 & Trueperaceae & $B-42$ \\
\hline 0.01553 & Spirochaetaceae & Treponema & 0.001035 & Actinomycetaceae & Mobiluncus \\
\hline 0.014495 & Enterobacteriaceae & Providencia & 0.001035 & Actinopolysporaceae & Actinopolyspora \\
\hline 0.014495 & Enterobacteriaceae & Salmonella & 0.001035 & Bogoriellaceae & Georgenia \\
\hline 0.014495 & Xanthomonadaceae & Lysobacter & 0.001035 & Kineosporiaceae & Kineococcus \\
\hline 0.01346 & Rhodobacteraceae & Rubellimicrobium & 0.001035 & Microbacteriaceae & Cryocola \\
\hline 0.01346 & Burkholderiaceae & Lautropia & 0.001035 & Micromonosporaceae & Actinoplanes \\
\hline 0.01346 & Halomonadaceae & Halomonas & 0.001035 & Micromonosporaceae & Catellatospora \\
\hline 0.01346 & Xanthomonadaceae & Luteimonas & 0.001035 & Micromonosporaceae & Virgisporangium \\
\hline 0.012424 & Lachnospiraceae & Catonella & 0.001035 & Thermomonosporaceae & Actinomadura \\
\hline 0.012424 & Comamonadaceae & Variovorax & 0.001035 & Yaniellaceae & Yaniella \\
\hline 0.011389 & Rubrobacteraceae & Rubrobacter & 0.001035 & Fimbriimonadaceae & Fimbriimonas \\
\hline 0.011389 & Porphyromonadaceae & Paludibacter & 0.001035 & Cytophagaceae & Emticicia \\
\hline
\end{tabular}




\begin{tabular}{|c|c|c|c|c|c|}
\hline 0.011389 & Flavobacteriaceae & Gramella & 0.001035 & Cytophagaceae & Larkinella \\
\hline 0.011389 & Weeksellaceae & Wautersiella & 0.001035 & Cytophagaceae & Sporocytophaga \\
\hline 0.011389 & Paenibacillaceae & Paenibacillus & 0.001035 & Weeksellaceae & Elizabethkingia \\
\hline 0.011389 & Exiguobacteraceae & Exiguobacterium & 0.001035 & Sphingobacteriaceae & Mucilaginibacter \\
\hline 0.011389 & Bradyrhizobiaceae & Bosea & 0.001035 & Sphingobacteriaceae & Pedobacter \\
\hline 0.011389 & Sinobacteraceae & Steroidobacter & 0.001035 & Balneolaceae & Balneola \\
\hline 0.010353 & Gordoniaceae & Gordonia & 0.001035 & Balneolaceae & $K S A 1$ \\
\hline 0.010353 & Micrococcaceae & Arthrobacter & 0.001035 & Ardenscatenaceae & Ardenscatena \\
\hline 0.010353 & Nocardioidaceae & Nocardioides & 0.001035 & Phormidiaceae & Planktothrix \\
\hline 0.010353 & Planococcaceae & Staphylococcus & 0.001035 & Bacillaceae & Marinococcus \\
\hline 0.010353 & Clostridiaceae & $S M B 53$ & 0.001035 & Paenibacillaceae & Cohnella \\
\hline 0.010353 & Lachnospiraceae & Shuttleworthia & 0.001035 & Planococcaceae & Bacillus \\
\hline 0.010353 & Bdellovibrionaceae & Bdellovibrio & 0.001035 & Planococcaceae & Planococcus \\
\hline 0.010353 & Xanthomonadaceae & Pseudoxanthomonas & 0.001035 & Aerococcaceae & Granulicatella \\
\hline 0.009318 & Rikenellaceae & Rikenella & 0.001035 & Aerococcaceae & Marinilactibacillus \\
\hline 0.009318 & Paraprevotellaceae & $C F 231$ & 0.001035 & Clostridiaceae & Proteiniclasticum \\
\hline 0.009318 & Ruminococcaceae & Anaerotruncus & 0.001035 & Peptococcaceae & Desulfotomaculum \\
\hline 0.009318 & Tissierellaceae & Finegoldia & 0.001035 & Peptostreptococcaceae & Clostridium \\
\hline 0.009318 & Tissierellaceae & Gallicola & 0.001035 & Veillonellaceae & Acidaminococcus \\
\hline 0.009318 & Rhizobiaceae & Agrobacterium & 0.001035 & Veillonellaceae & Megamonas \\
\hline 0.009318 & Acetobacteraceae & Commensalibacter & 0.001035 & Acidaminobacteraceae & Fusibacter \\
\hline 0.009318 & Campylobacteraceae & Arcobacter & 0.001035 & Tissierellaceae & Helcococcus \\
\hline 0.009318 & Legionellaceae & Legionella & 0.001035 & Erysipelotrichaceae & Clostridium \\
\hline 0.009318 & Halomonadaceae & Kushneria & 0.001035 & Erysipelotrichaceae & Sharpea \\
\hline 0.009318 & Vibrionaceae & Vibrio & 0.001035 & Caulobacteraceae & Asticcacaulis \\
\hline 0.009318 & Opitutaceae & Opitutus & 0.001035 & Caulobacteraceae & Nitrobacteria \\
\hline 0.008283 & Aerococcaceae & Abiotrophia & 0.001035 & Caulobacteraceae & Phenylobacterium \\
\hline 0.008283 & Lachnospiraceae & Moryella & 0.001035 & Kiloniellaceae & Thalassospira \\
\hline 0.008283 & Ruminococcaceae & Butyricicoccus & 0.001035 & Bradyrhizobiaceae & Bradyrhizobium \\
\hline 0.008283 & Rhodobacteraceae & Amaricoccus & 0.001035 & Phyllobacteriaceae & Aminobacter \\
\hline 0.008283 & Oxalobacteraceae & Cupriavidus & 0.001035 & Phyllobacteriaceae & Aquamicrobium \\
\hline 0.008283 & Enterobacteriaceae & Enterobacter & 0.001035 & Rhizobiaceae & Kaistia \\
\hline 0.008283 & Chthoniobacteraceae & Candidatus Xiphinematobacter & 0.001035 & Rhodospirillaceae & Magnetospirillum \\
\hline 0.007247 & Nocardioidaceae & Pimelobacter & 0.001035 & Rickettsiaceae & Rickettsia \\
\hline 0.007247 & Rikenellaceae & $A F 12$ & 0.001035 & mitochondria & Arabidopsis \\
\hline 0.007247 & Planococcaceae & Sporosarcina & 0.001035 & mitochondria & Citrullus \\
\hline 0.007247 & Lactobacillaceae & Pediococcus & 0.001035 & Sphingomonadaceae & Sandaracinobacter \\
\hline 0.007247 & Coxiellaceae & Coxiella & 0.001035 & Alcaligenaceae & Alcaligenes \\
\hline 0.006212 & Cellulomonadaceae & Actinotalea & 0.001035 & Alcaligenaceae & Rhodospirillum \\
\hline 0.006212 & Nocardioidaceae & Propionicimonas & 0.001035 & Comamonadaceae & Rubrivivax \\
\hline 0.006212 & Bifidobacteriaceae & Gardnerella & 0.001035 & Methylophilaceae & Methylobacillus \\
\hline 0.006212 & Cryomorphaceae & Fluviicola & 0.001035 & Desulfarculaceae & Desulfarculus \\
\hline 0.006212 & Lachnospiraceae & Roseburia & 0.001035 & HТCС 2188 & HTCC \\
\hline 0.006212 & Acidaminobacteraceae & Guggenheimella & 0.001035 & Idiomarinaceae & Pseudidiomarina \\
\hline 0.006212 & Planctomycetaceae & Planctomyces & 0.001035 & Chromatiaceae & Alkalimonas \\
\hline 0.006212 & Erythrobacteraceae & Citromicrobium & 0.001035 & Halomonadaceae & Chromohalobacter \\
\hline 0.006212 & Sphingomonadaceae & Sphingopyxis & 0.001035 & Verrucomicrobiaceae & Luteolibacter \\
\hline 0.006212 & Comamonadaceae & Comamonas & 0.001035 & Verrucomicrobiaceae & Verrucomicrobium \\
\hline 0.006212 & Oxalobacteraceae & Janthinobacterium & 0.001035 & Chthoniobacteraceae & DA101 \\
\hline 0.006212 & Aeromonadaceae & Aeromonas & & & \\
\hline
\end{tabular}




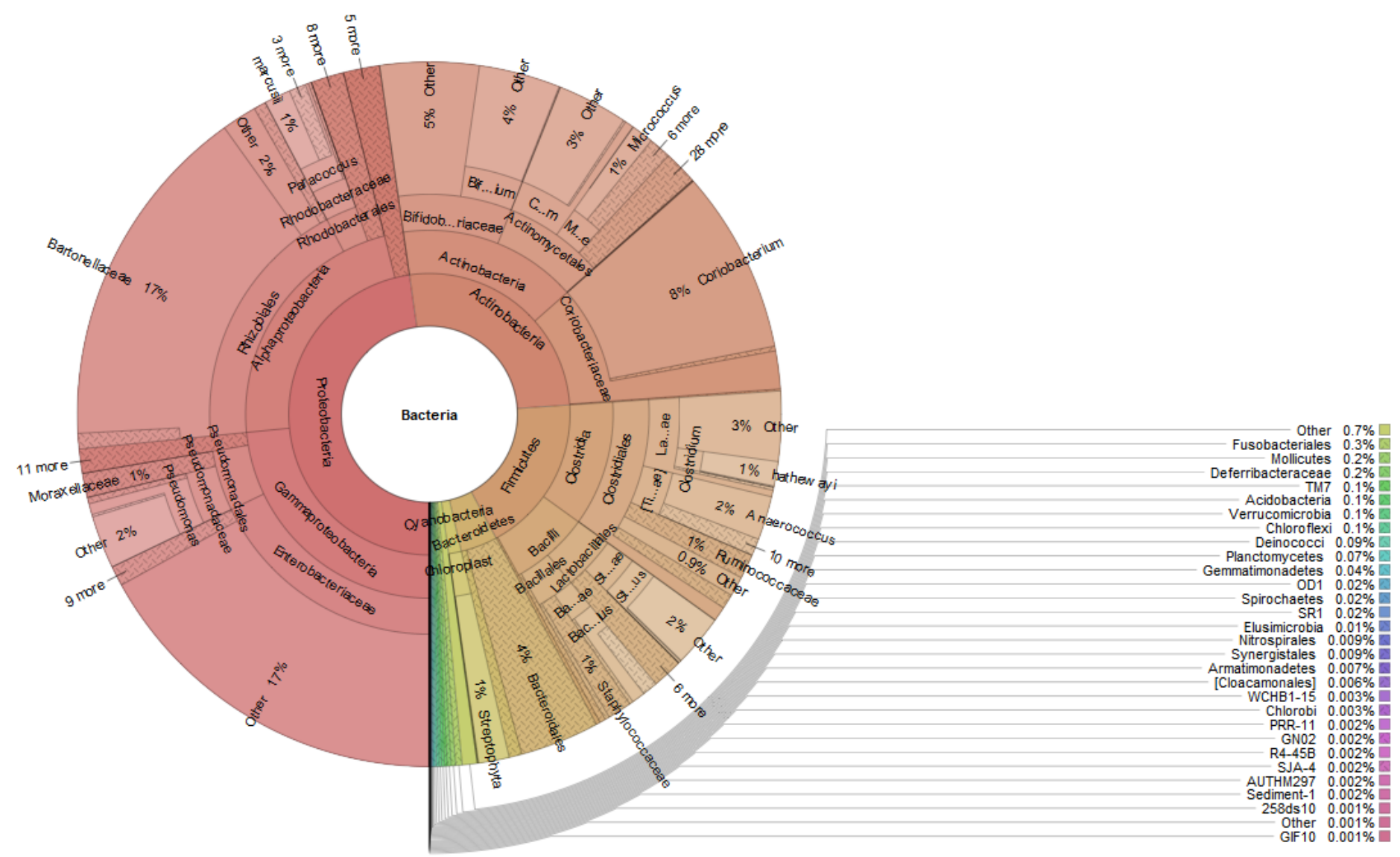

Figure 4 Pie chart showing the distribution of species under Genus level classification

\section{DISCUSSION}

Illumina MiSeq technology was applied to unravel the bacterial composition of the gut of Dysdercus cingulatus by analyzing the V3 and V4 hyper variable regions of 16S rRNA. In this study, we identified Proteobacteria, Actinobacteria, and Firmicutes as the three major phyla presenting the gut of $D$. cingulatus. The study shows that Bartonella was the most abundant genus present in the gut of the pest. Bartonella is a collection of gram-negative bacteria belonging to a single genus of the family Bartonellaceae. These are human pathogens transmitted by sand flies, ticks, and fleas. Bartonella was identified at species level as Bartonella bacilliformis that is known to have a genetic relationship with plant symbionts such as Rhizobium meliloti, a nitrogen fixing bacteria (Ihler, 1996). The second largest genus in the gut was an unidentified taxon belonging to the family Enterobacteriaceae followed by genus Coriobacterium from family Coriobacteriaceae whose members provide vitamin B to their insect host (H. Salem et al., 2014).

Bifidobacteria was another abundant bacterial genus identified in the gut of $D$. cingulatus. Bifidobacteria are fermentative organisms that are present in the gut of various animals and human. They are capable of metabolizing carbohydrate and other types of glycans present in the gut of animals (Pokusaeva, Fitzgerald, \& Van Sinderen, 2011). Bifidobacteria are also known to be present in insects. Nearly $2-8.4 \%$ of the total population was identified as Bifidobacteria in the gut of honey bees and homogenous in composition, whereas bifidobacterial population in wasps was heterogeneous (Mrázek, Štrosová, Fliegerová, Kott, \& Kopečný, 2008). Here, we report two different species of Bifidobacteria namely Bifidobacterium asteroids and Bifidobacterium longum. In Apis mellifera, Bifidobacterium asteroids PRL2011can perform respiratory metabolism (Bottacini et al., 2012).

Corynebacterium is a pantothenic acid producing bacteria in the gut of Triatoma infestans (Durvasula et al., 2008). In our study, we identified various species of Corynebacterium namely $C$. matruchotii, $C$. matruchotii, C. tuscaniense, $C$. glaucum, $C$. kroppenstedtii, $C$. suicordis, $C$. bovis, $C$. timonense, $C$. durum, $C$. coyleae, and C. riegelii.

Klebsiella represents $3.5 \%$ of gut Microbiome of D. cingulatus. Klebsiella isolated from guts of larvae of Aularches miliaris, Propylea quatuordecimpunctata and nymphal stage of Oxya veloxis reported to be a cellulose degrading bacterium (Shil, Mojumder, Sadida, Uddin, \& Sikdar, 2014). Similarly, Klebsiella isolated from Bombyx mori also degrades polysaccharides in mulberry leaves. Our study identified Klebsiella pneumonia in the gut of $D$. cingulatus. Isolation and characterization of $K$. pneumonia confirmed cellulolytic and xylanolytic properties in the gut of Bombyx mori (Anand et al., 2010).
Two species of Streptococcus namely S. mitis and S. parauberis were identified in the gut of D. cingulatus. S. mitis had also been reported in the gut of pine engraver Ips pini that colonizes red pine (Jr et al., 2007). Similarly, Paracoccus aestuarii and Paracoccus marcusii from family Rhodobacteraceae, and Anaerococcus hydrogenalis, Anaerococcus lactolyticus, and Anaerococcus prevotii from family Tissierellaceae were also identified in $D$. cingulatus.

Bacillus is one of the most common bacterial species found in the guts of all the insects. Bacillus species isolated from guts of larvae of five different families of wood-feeding Coleoptera collected from tropical forests of Costa Rica possessed $\beta$-glucosidase, $\beta$-xylanase, and cellobiose hydrolase activities (Rojas-Jiménez \& Hernández, 2015). In D. cingulatus, Bacillus makes $1.48 \%$ of the gut bacterial population. Bacillus species identified in our study were B. subtilis, B. niacin, $B$. firmus, B. badius, B. licheniformis, and B. megaterium.

Micrococcaceae is another frequently isolated family of bacteria from the mid guts of various insect species. Three different genera identified in this study were Rothia, Arthrobacter, Micrococcus and Kocuria (Rizzi et al., 2013). The most abundant bacterial species identified from this family were $R$. mucilaginosa and $R$. dentocariosa. In humans, Rothia has been identified to colonize the upper gastro-intestinal tract and helps in the degradation of gluten.

Similarly, Micrococcus lutens is an inhabitant of the human mouth, pharynx, and respiratory tract. Member of genus Micrococcus is also found in insects. Gut bacterial composition study had identified M. lutens as one of the bacteria present in the pre-pupae and adult stages of sub cortical beetle, Agrilus plannipennis (Vasanthakumar, Handelsman, Schloss, Bauer, \& Raffa, 2008). Other two bacterial species identified in this study were Arthrobacter keyseri, Kocuria rosea.

Other important genera present in D. cingulatus are Propionibacterium Prevotella, Staphylococcus, Acinetobacter, Enterobacter, and Enterococcus. We identified three species of Propionibacterium namely $P$. acnes, $P$. granulosum and $P$. acidifaciens. Among them, $P$. acnes is abundant compared to other species. Propionibacterium spp. are common soil inhabitants with different metabolic characteristics that may be advantageous to their insect hosts. Some species of Propionibacterium are lipolytic (Jarvis, Strömpl, Moore, \& Thiele, 1998). Similarly, Acinetobacter spp. have been correlated to phosphate storage (Rustrian, Delgenes, \& Moletta, 1997).

Staphylococcus, an important genus of Firmicutes is present in most of the insects and non- pathogenic. Their abundance and variety differ with different food habits. The comparative study of gut bacteria present in Dastarcus helophoroides fed on different artificial diets identified Staphylococcus as one of the predominant genera in the gut (Zhang, He, $\& \mathbf{L i}, \mathbf{2 0 1 4})$. Gut bacteria identified at the species level in nine different species of Australian termites 
demonstrated the presence of Staphylococcus in three different species from family Termitidae (Eutick, O'Brien, \& Slaytor, 1978). In D. cingulatus we identified four different species of Staphylococcus namely $S$. sciuri, $S$. epidermidis, S. hominis, S. pasteuri, and S. succinus.

Enterococci belonging to phylum Firmicutes are known to harbor a large variety of insects which includes beetles, termites, flies and worms. They are mostly present in species feeding on succulent parts of plants and nectar. E. faecalis and E. faecium are the predominant species but other species appear to lesser extent (Martin \& Mundt, 1972). In Harpalus Pensylvanicus, E. faecalis increases the seed consumption by providing the digestive enzymes to their host (Schmid, Lehman, Brözel, \& Lundgren, 2014). E. durans is the only bacteria species that was identified from the genus Enterococcus.

$E$. durans isolated from the gut of $O$. velox, $A$. miliaris and $P$. quatuordecimpunctata was identified as cellulolytic bacteria along with bacteria from other genera. Genus Anaerococcus is a butyrate-producing species. The seed-parasitic wasp of genus Megastigmus, Anaerococcus was the major Firmicute (Paulson, Aderkas, \& Perlman, 2014). Our study also identified Anaerococcus spp. A. Prevotti, A. lactolyticus, and A. Hyarogenati in the gut of $D$. cingulatus with a total abundance of $2 \%$. Butyrate and energy molecule producing other bacteria identified in the gut of $D$. cingulatus were Butyrivibrio fibrisolvens, Butyrivibrio hungatei, Dorea formicigenerans, Roseburia inulinivorans, Roseburia faecis, and Roseburia cecicola from the family Lachnospiraceae (Titus \& Ahearn, 1988)

\section{CONCLUSION}

Dysdercus cingulatus (Hemiptera: Pyrrhocoridae) is a cotton pest that has noxious effect on the cotton seeds and cotton lint. Bacterial community colonizing the gut of this pest was identified using 16S rRNA Metagenomics sequencing technique. The bacterial species were identified using QIIME. The analysis shows the presence of bacterial species from genera Coriobacterium, Bifidobacterium, Corynebacterium, Pseudomonas and many others. These bacteria are highly important in their own self because of their vital bioprocessing capabilities. This study will facilitate further in identifying specific roles played by some of these major bacteria identified in the gut of $D$. cingulatus. Identification of such bacterial species that are important for the survival of the insect could be used to initiate new strategies for pest management.

\section{REFERENCES}

Ahmad, I., \& Schaefer, C. W. (1987). Food plant and feeding biology of the Pyrrhocoridae (Hemiptera). Phytophaga, 1, 75-92.

Anand, A. A. P., Vennison, S. J., Sankar, S. G., Prabhu, D. I. G., Vasan, P. T., Raghuraman, T., ... Vendan, S. E. (2010). Isolation and characterization of bacteria from the gut of Bombyx mori that degrade cellulose, xylan, pectin and starch and their impact on digestion. Journal of Insect Science (Online), 10 https://doi.org/10.1673/031.010.10701

Bartram, A. K., Lynch, M. D. J., Stearns, J. C., Moreno-Hagelsieb, G., \& Neufeld, J. D. (2011). Generation of multimillion-sequence 16S rRNA gene libraries from complex microbial communities by assembling paired-end Illumina reads. Applied and Environmental Microbiology, 77(11), 3846-3852 https://doi.org/10.1128/AEM.02772-10

Bennett, A. E. (2013). Can plant-microbe-insect interactions enhance or inhibit the spread of invasive species? Functional Ecology, 27(3), 661-671 https://doi.org/10.1111/1365-2435.12099

Bottacini, F., Milani, C., Turroni, F., Sánchez, B., Foroni, E., Duranti, S., ..

Ventura, M. (2012). Bifidobacterium asteroides PRL2011 Genome Analysis Reveals Clues for Colonization of the Insect Gut. PLoS ONE, 7(9), 1-14 https://doi.org/10.1371/journal.pone.0044229

Caporaso, J. G., Kuczynski, J., Stombaugh, J., Bittinger, K., Bushman, F. D. Costello, E. K., ... Walters, W. a. (2010). QIIME allows analysis of highthroughput community sequencing data. Nature Methods, 7(5), 335-336. https://doi.org/10.1038/nmeth.f.303.0IIME

Durvasula, R. V, Sundaram, R. k, Kirsch, P., Hurwitz, I., Crawford, C. V Dotson, E., \& Beard, C. B. (2008). Genetic transformation of a Corynebacterial symbiont from the Chagas disease vector Triatoma infestans. Expermental Parasitology, $119(1)$ https://doi.org/10.1016/i.exppara.2007.12.020.Genetic

Eutick, M. L., O'Brien, R. W., \& Slaytor, M. (1978). Bacteria from the gut of Australian termites. Applied and Environmental Microbiology, 35(5), 823-828.

Huse, S. M., Dethlefsen, L., Huber, J. A., Welch, D. M., Relman, D. A., \& Sogin, M. L. (2008). Exploring microbial diversity and taxonomy using SSU rRNA hypervariable tag sequencing. PLoS Genetics, 4(11). https://doi.org/10.1371/journal.pgen.1000255

Ihler, G. M. (1996). Bartonella bacilliformis: dangerous pathogen slowly emerging from deep background. FEMS Microbiology Letters, 144(1), 1-11 https://doi.org/10.1016/0378-1097(96)00307-2

Janda, J. M., \& Abbott, S. L. (2007). 16S rRNA Gene Sequencing for Bacterial Identification in the Diagnostic Laboratory: Pluses, Perils, and Pitfalls. Journal of
Clinical Microbiology, 45(9), 2761-2764. https://doi.org/10.1128/JCM.01228 07

Jarvis, G. N., Strömpl, C., Moore, E. R. B., \& Thiele, J. H. (1998). Isolation and Characterisation of Obligately Anaerobic, Lipolytic Bacteria from the Rumen of Red Deer. Systematic and Applied Microbiology, 21(1), 135-143. https://doi.org/10.1016/S0723-2020(98)80017-9

Jr, I. D., Vasanthakumar, A., Burwitz, B. J., Schloss, P. D., Klepzig, K. D., Handelsman, J., \& Raffa, K. F. (2007). Composition of the bacterial community in the gut of the pine engraver, Ips pini (Say) (Coleoptera) colonizing red pine. Symbiosis, 43, 97-104.

Kress, W. J., \& Erickson, D. L. (2008). DNA barcodes: Genes, genomics, and bioinformatics. Proceedings of the National Academy of Sciences of the United States of America, 105(8), 2761-2762. https://doi.org/10.1073/pnas.0800476105

Magoč, T., \& Salzberg, S. L. (2011). FLASH: Fast length adjustment of short reads to improve genome assemblies. Bioinformatics, 27(21), 2957-2963. https://doi.org/10.1093/bioinformatics/btr507

Martin, J. D., \& Mundt, J. O. (1972). Enterococci in insects. Applied Microbiology, 24(4), 575-580.

Maxwell-Lefroy, H. (1906). Indian insect pests. Calcutta: Office of the Superintendent of Government Printing.

Mrázek, J., Štrosová, L., Fliegerová, K., Kott, T., \& Kopečný, J. (2008). Diversity of insect intestinal microflora. Folia Microbiologica, 53(3), 229-233 https://doi.org/10.1007/s12223-008-0032-Z

Paulson, A. R., Aderkas, P. Von, \& Perlman, S. J. (2014). Bacterial associates of seed-parasitic wasps (Hymenoptera: Megastigmus) Bacterial associates of seedparasitic wasps (Hymenoptera: Megastigmus), 1-15 https://doi.org/10.1186/s12866-014-0224-4

Pokusaeva, K., Fitzgerald, G. F., \& Van Sinderen, D. (2011). Carbohydrate metabolism in Bifidobacteria. Genes and Nutrition, 6(3), 285-306. https://doi.org/10.1007/s12263-010-0206-6

Rizzi, A., Crotti, E., Borruso, L., Jucker, C., Lupi, D., Colombo, M., \& Daffonchio, D. (2013). Characterization of the bacterial community associated with larvae and adults of anoplophora chinensis collected in Italy by culture and culture-independent methods. BioMed Research International, 2013. https://doi.org/10.1155/2013/420287

Rojas-Jiménez, K., \& Hernández, M. (2015). Isolation of fungi and bacteria associated with the guts of tropical wood-feeding coleoptera and determination of their lignocellulolytic activities. International Journal of Microbiology, 2015 https://doi.org/10.1155/2015/285018

Rustrian, E., Delgenes, J. P., \& Moletta, R. (1997). Phosphate release and uptake by pure cultures of Acinetobacter sp.: Effect of the volatile fatty acids concentration. Current Microbiology, 34(1), 43-48. https://doi.org/10.1007/s002849900142

Salem, H., Bauer, E., Strauss, A. S., Vogel, H., Marz, M., \& Kaltenpoth, M (2014). Vitamin supplementation by gut symbionts ensures metabolic homeostasis in an insect host. Proceedings of the Royal Society B: Biological
Sciences, 281(1796),
20141838-20141838.

https://doi.org/10.1098/rspb.2014.1838

Salem, H., Kreutzer, E., Sudakaran, S., \& Kaltenpoth, M. (2012). Actinobacteria as essential symbionts in firebugs and cotton stainers (Hemiptera, Pyrrhocoridae). Environmental Microbiology, 15(7), 1956-1968. https://doi.org/10.1111/1462 2920.12001

Schmid, R. B., Lehman, R. M., Brözel, V. S., \& Lundgren, J. G. (2014). An Indigenous Gut Bacterium, Enterococcus faecalis (Lactobacillales: Enterococcaceae), Increases Seed Consumption by Harpalus pensylvanicus (Coleoptera: Carabidae). Florida Entomologist, 97(2), 575-584. https://doi.org/10.1653/024.097.0232

Shil, R. K., Mojumder, S., Sadida, F. F., Uddin, M., \& Sikdar, D. (2014) Isolation and identification of cellulolytic bacteria from the gut of three phytophagus insect species. Brazilian Archives of Biology and Technology, 57(6), 927-932. https://doi.org/10.1590/S1516-8913201402620

Shivas, R. G., Smith, M. W., Marney, T. S., Newman, T. K., Hammelswang, D. L., Cooke, A. W., ... Pascoe, I. G. (2005). First record of Nematospora coryli in Australia and its association with dry rot of Citrus. Australasian Plant Pathology, 34(1), 99-101. https://doi.org/10.1071/AP04075

Singh., H. (1924). On the Anatomy and Bionomics of the Bed Cotton Bug, Dysdercus cingulatus (Fabr.). Jl. \& Proc. Asiatic Soc. Bengal. Calcutta, 19, 1542 . Retrieved from https://archive.org/stream/mobot31753002183942/mobot $31753002183942 \mathrm{dj}$ vu.txt

Srinivasan, R., Karaoz, U., Volegova, M., MacKichan, J., Kato-Maeda, M. Miller, S., ... Lynch, S. V. (2015). Use of 16S rRNA gene for identification of a broad range of clinically relevant bacterial pathogens. PLoS ONE, 10(2), 1-22 https://doi.org/10.1371/journal.pone.0117617

Takahashi, S., Tomita, J., Nishioka, K., Hisada, T., \& Nishijima, M. (2014) Development of a prokaryotic universal primer for simultaneous analysis of Bacteria and Archaea using next-generation sequencing. PLoS ONE, 9(8). https://doi.org/10.1371/journal.pone.0105592 
Taylor, C. M., Coffey, P. L., DeLay, B. D., \& Dively, G. P. (2014). The importance of gut symbionts in the development of the brown marmorated stink
bug,
Halyomorpha
halys
(Stål).
PLoS
ONE,
$9(3)$

Titus, E., \& Ahearn, G. a. (1988). Short-chain fatty acid transport in the intestine of a herbivorous teleost. The Journal of Experimental Biology, 135, 77-94.

Vasanthakumar, A., Handelsman, J., Schloss, P. D., Bauer, L. S., \& Raffa, K. F

(2008). Gut microbiota of an invasive subcortical beetle, Agrilus planipennis Fairmaire, across various life stages. Environmental Entomology, 37(5), 1344 1353. https://doi.org/10.1603/0046-225X(2008)37

Xiang, H., Xie, L., Zhang, J., Long, Y. H., Liu, N., Huang, Y. P., \& Wang, Q (2012). Intracolonial differences in gut bacterial community between worker and soldier castes of Coptotermes formosanus. Insect Science, 19(1), 86-95. https://doi.org/10.1111/j.1744-7917.2011.01435.x

Zhang, Z. Q., He, C., \& Li, M. L. (2014). Analysis of intestinal bacterial community diversity of adult Dastarcus helophoroides. Journal of Insect Science, 14(114), 1-13. https://doi.org/10.1093/jis/14.1.114 